\title{
The nucleus basalis (Ch4) in the alcoholic Wernicke-Korsakoff syndrome: reduced cell number in both amnesic and non-amnesic patients
}

\author{
K M Cullen, G M Halliday, D Caine, J J Kril
}

\section{Department of Pathology, Neuropathology Division, The University of Sydney 2006, NSW 2006, \\ Australia \\ K M Cullen \\ G M Halliday \\ J J Kril \\ Prince of Wales Medical Research Institute, High Street, Randwick, NSW 2031, Australia \\ G M Halliday}

Neuropsychology Unit, Royal Prince Alfred

Hospital,

Camperdown, NSW

2050, Australia

D Caine

Correspondence to:

Dr K M Cullen,

Neuroscience Unit, School of Biological Sciences A08, The University of Sydney,

Sydney, NSW 2006,

Australia.

Received 27 November 1996 and in revised form 27 January 1997

Accepted 12 February 1997

\begin{abstract}
Background-The cholinergic nucleus basalis (Ch4) is an exclusive site of neurofibrillary degeneration in alcoholic patients with Wernicke's encephalopathy. Aim-To test the hypothesis that the loss of $\mathrm{Ch} 4$ neurons contributes to the memory disorder, Korsakoff's psychosis, commonly seen in Wernicke's encephalopathy. Methods-Magnocellular basal forebrain neurons were quantified in alcoholic patients with Wernicke's encephalopathy, both with and without Korsakoff's psychosis, and neurologically asymptomatic alcoholic and non-alcoholic controls. Because amnesic and non-amnesic patients with Wernicke's encephalopathy share common periventricular lesions, both thiamine deficient groups as well as alcoholic patients with no neurological complications were included to determine the lesion specific to memory impairment.
\end{abstract}

Results-Ch4 cell number did not differ significantly between alcoholic and nonalcoholic controls and there was no correlation between cell number and lifetime alcohol intake. However, $\mathrm{Ch} 4$ cell number in all groups was significantly correlated with the volume of its major projection target, the cerebral cortex. Ch4 cell number in the non-amnesic Wernicke's encephalopathy group was significantly below controls $(24 \%)$, with cell number in patients with Korsakoff's psychosis $21 \%$ below controls. There was considerable overlap in cell number between groups. On discriminant analysis, there was significantly greater cell loss in three nonamnesic patients with Wernicke's encephalopathy than in some patients with Korsakoff's psychosis. The nonamnesic patient with the greatest cell loss was impaired on attentional tasks. Conclusion-Whereas neurons in the nucleus basalis are at risk in thiamine deficient alcoholic patients, cell loss is minor and does not account for the profound memory disorder.

(F Neurol Neurosurg Psychiatry 1997;63:315-320)

Keywords: Wernicke's encephalopathy; Korsakoff's psychosis; nucleus basalis; memory; alcohol
Although there are several comprehensive descriptions of the neuropathology of Wernicke's encephalopathy, ${ }^{1-3}$ definitive anatomical substrates for the associated amnesic syndrome, Korsakoff's psychosis, have not been identified. We have previously shown that the cholinergic nucleus basalis (Ch4) is an exclusive site of neurofibrillary degeneration in thiamine deficient alcoholic patients. ${ }^{4}$ These findings prompted us to investigate the degree of Ch4 cell loss in association with the memory disorder Korsakoff's psychosis.

The notion that cholinergic deficits can result in memory dysfunction has been extensively evaluated in several animal and pharmacological models as well as in neurodegenerative diseases, most notably Alzheimer's disease. A role for the $\mathrm{Ch} 4$ in memory has been supported by a previous study of patients with Korsakoff's psychosis correlating loss of magnocellular basal forebrain neurons to memory dysfunction. ${ }^{56}$ To date this hypothesis remains largely unchallenged. The present study compared the Ch4 in patients with Wernicke's encephalopathy and those with additional Korsakoff's psychosis with age matched alcoholic and non-alcoholic controls to test the hypothesis that amnesic patients have a common anatomical lesion in the $\mathrm{Ch} 4$. As the $\mathrm{Ch} 4$ provides most of the cholinergic innervation to the cortex, ${ }^{7}$ cell number was also compared with the brain atrophy reported in alcoholic patients. $^{8}$

\section{Materials and methods}

CASE SELECTION

The present work is part of a broader investigation on memory specific lesions. ${ }^{89}$ All cases (table) were selected and classified retrospectively. The cases were evaluated as part of an assessment of operational criteria for the classification of chronic alcoholic patients without neurological abnormalities and alcoholic patients with Wernicke's encephalopathy or with Wernicke's encephalopathy and Korsakoff's psychosis. ${ }^{9}$ Written consent was obtained for hospital necropsies and the study was approved by the human ethics committee of the University of Sydney and Royal Prince Alfred Hospital under the New South Wales Transplantation and Anatomy Act. Details of diet and alcohol consumption were obtained from clinical records and written and telephone communication with general practition- 


\begin{tabular}{|c|c|c|c|c|c|c|c|}
\hline Case & Age (y) & $\begin{array}{l}\text { Sex } \\
(M / F)\end{array}$ & $\begin{array}{l}\text { Delay pm } \\
\text { (h) }\end{array}$ & $\begin{array}{l}\text { Duration of } \\
\text { disease }\end{array}$ & $\begin{array}{l}\text { Ch4 cell } \\
\text { number }\end{array}$ & $\begin{array}{l}\text { Ch4 mean cell } \\
\text { diameter }(\mu m)\end{array}$ & $\begin{array}{l}\text { Volume cerebral } \\
\text { hemispheres }\left(\mathrm{cm}^{3}\right)\end{array}$ \\
\hline $\mathrm{C} 1$ & 20 & $M$ & 6 & - & 415420 & $32.1(4.4)$ & 1474 \\
\hline $\mathrm{C} 2$ & 37 & M & 24 & - & 323000 & $31.0(3.7)$ & 1295 \\
\hline C3 & 50 & $\mathrm{~F}$ & 6 & - & 444100 & $31.0(4.0)$ & 1115 \\
\hline $\mathrm{C} 4$ & 60 & $\mathrm{~F}$ & 2 & - & 311100 & $30.1(4.1)$ & 998 \\
\hline C5 & 78 & $\mathrm{~F}$ & 24 & - & 312150 & $30.3(3.8)$ & 957 \\
\hline C6 & 80 & M & 22 & - & 252320 & $30.2(3.7)$ & 971 \\
\hline $\mathrm{C} 7$ & 88 & $\mathrm{~F}$ & 19 & - & 206910 & $29.8(4.0)$ & 1007 \\
\hline C8 & 92 & $\mathrm{~F}$ & 24 & - & 252480 & $29.2(2.9)$ & 930 \\
\hline A1 & 60 & M & 16 & - & 346876 & $30.2(1.7)$ & 1110 \\
\hline $\mathrm{A} 2$ & 61 & M & 12 & - & 350610 & $31.0(4.1)$ & 937 \\
\hline A3 & 66 & M & 10 & - & 300600 & 30.1 (3.8) & 1019 \\
\hline A4 & 71 & M & 12 & - & 381510 & $30.2(3.7)$ & 1182 \\
\hline A5 & 66 & M & 8 & - & 339765 & $31.3(3.0)$ & 1424 \\
\hline WE1 & 51 & M & 30 & 2-3 Days & 340890 & $31.1(3.6)$ & 1071 \\
\hline WE2 & 59 & $\mathrm{~F}$ & 24 & $11 \mathrm{y}$ & 315840 & $32.1(4.1)$ & 978 \\
\hline WE3 & 59 & M & 12 & $\sim 2 \mathrm{y}$ & 276060 & $30.6(4.0)$ & 1090 \\
\hline WE4 & 57 & M & 72 & $\sim 2 \mathrm{y}$ & 231300 & $30.5(3.5)$ & 978 \\
\hline WE5 & 52 & M & 37 & $>7 \mathrm{y}$ & 202410 & $28.4(3.5)$ & 1017 \\
\hline KP1 & 60 & M & 23 & $>9 \mathrm{y}$ & 332490 & $32.7(3.1)$ & 952 \\
\hline $\mathrm{KP} 2$ & 60 & M & 15 & $22 \mathrm{y}$ & 302690 & $31.5(3.9)$ & 1016 \\
\hline KP3 & 46 & M & 9 & $>10 y$ & 266400 & $33.2(3.1)$ & 1120 \\
\hline KP4 & 78 & $\mathrm{~F}$ & 28 & $>30 \mathrm{y}$ & 256680 & $28.6(2.7)$ & 1245 \\
\hline
\end{tabular}

ers as well as from written questionnaires distributed to relatives of the patients. Most alcoholic patients (A1-A5, KP1, KP2, and KP4; table) were seen in the neuropsychology unit at Royal Prince Alfred Hospital; cases KP3 and WE4 were seen at the Royal Brisbane Hospital.

Strict exclusion criteria were employed for the purposes of this study: cases were not included if there was evidence of stroke, head injury, or Alzheimer type cortical pathology (neuritic plaques and neurofibrillary tangles), if details of alcohol and thiamine status could not be ascertained, or if time from death to postmortem was greater than 72 hours. All cases of Wernicke's encephalopathy studied, including those with Korsakoff's psychosis, had documentation in medical records of least two of the classic triad of symptoms, although most had evidence of all three. Our previous work has shown that neurological evidence of Wernicke's encephalopathy was present in most (94\%) cases of Korsakoff's psychosis, with Wernicke's encephalopathy neuropathology in alcoholic Korsakoff's psychosis a universal finding. ${ }^{9}$

The mamillary bodies in all cases with chronic Wernicke's encephalopathy were small and brown, with capillary proliferation, neuronal loss, and gliosis, consistent with chronic Wernicke's encephalopathy. Cerebellar vermal atrophy was noted in all alcoholic patients with chronic Wernicke's encephalopathy, including those with Korsakoff's psychosis. Four cases (WE2, WE3, WE4, and KP2) had pathologically confirmed liver disease, with case WE4 showing evidence of hepatic encephalopathy. Brainstem, hippocampal, and hypothalamic pathology has been previously reported in all alcoholic cases excluding case WE4 (brainstem) and KP4 and WE2 (hypothalamus). All cases except KP1 and KP2 have been included in previous reports on the basal forebrain. ${ }^{4} 10$

Non-alcoholic controls $(\mathrm{n}=8$, mean age 63 (SD 26) years) consumed less than $20 \mathrm{~g}$ alcohol per day and had no psychological, neurological, or neuropathological abnormalities and no evidence of thiamine deficiency. Age matched controls ( $\mathrm{n}=5$; C1-C5; mean age 49 (SD 22); postmortem delay 16 (SD 10) hours) were used for all group comparisons.

Alcoholic controls ( $\mathrm{n}=5$, mean age $=64$ (4); postmortem delay 12(3) hours) consumed greater than $80 \mathrm{~g}$ alcohol per day for at least 20 years and had no abnormal neurological signs. Medical histories and questionnaires distributed to family members indicated normal dietary intake and no memory disturbance. No neuropathology was detected in these cases, ${ }^{9}$ and there was no locus coeruleus or dorsal raphe nuclei cell loss.

Alcoholic patients with Wernicke's encephalopathy ( $\mathrm{n}=5$, mean age 55 (SD 4) years; postmortem delay 33 (SD 25) hours) consumed greater than $80 \mathrm{~g}$ (average intake $200 \mathrm{~g}$ ) alcohol per day for most of their adult lives. All were diagnosed during life on the basis of neurological signs (required the presence of at least two of the classic triad of signs) and were living independently at the time of death. Case WE1 had only one documented episode of Wernicke's encephalopathy and is designated here as having acute Wernicke's encephalopathy. All other cases of Wernicke's encephalopathy had persisting neurological signs and were classified as chronic. In addition to the classic signs of Wernicke's encephalopathy, cases WE2 and WE3 also showed frontal lobe signs (including abnormalities in planning, insight, or abstraction), cases WE3 and WE5 had mild intermittent memory dysfunction (defined as inability to remember at least two words in a four item test or mild impairment on more elaborate neuropsychological tests).

Alcoholic patients with Korsakoff's psychosis $(n=4$, mean age 61 (SD 17) years; postmortem delay 27 (SD11) hours) consumed greater than $80 \mathrm{~g}$ alcohol for most of their adult lives. Neurological signs of Wernicke's encephalopathy were reported in all four patients and a clinical diagnosis of Korsakoff's psychosis was made prospectively in all cases. All cases had been assessed in a study of diagnostic criteria for Korsakoff's psychosis ${ }^{9}$ and had pathology consistent with chronic Wernicke's encephalopathy. All cases had an amnesia defined as a 
stable and persistent inability to form new memories. Case KP2 also had frontal lobe signs.

\section{TISSUE PREPARATION}

The brains were removed at necropsy and fixed by immersion in $15 \%$ formalin ( $\mathrm{pH} 7.4$ ) for at least two weeks. Whole brain and cerebrum weights and volumes (by fluid displacement) were determined before and after fixation to assess shrinkage artefact ( $<5 \%$ in each case). The cerebrum was embedded in agar and sliced coronally on a rotary slicer at $3 \mathrm{~mm}$ intervals for macroscopic diagnosis and photographed at $1 \times$ magnification for volume determination. ${ }^{812}$ Cortical, brainstem, diencephalic, and cerebellar blocks were taken for routine histopathological evaluation. The basal forebrain from both hemispheres was removed and the blocks cryoprotected in Tris- $\mathrm{HCl}$ buffer $\mathrm{pH} 7.4$ containing $30 \%$ sucrose for two to three days, before serial sectioning at $50 \mu \mathrm{m}$ on a Leitz freezing microtome. Six series (1/15) of sections were stained with haematoxylin and eosin, a modified Bielschowsky silver stain, luxol fast blue, cresyl violet, and immunohistochemistry for tau and the calcium binding protein, calbindin-D 28k. Calbindin has a high degree of colocalisation with cholinergic specific markers, ${ }^{13}$ supporting its role as a specific marker for the $\mathrm{Ch} 4$ in the basal forebrain. For immunohistochemistry, calbindin-D 28k (1:200, Sigma, C8666) and tau (1:10 000, Sigma, T5530) binding were visualised using appropriate biotinylated secondary antibodies and the avidin-biotin peroxidase complex detection system (ABC kit, Vector Laboratories, Burlingame, CA, USA) with diaminobenzidine as the chromogen, described in detail previously. ${ }^{14}$

ANALYSIS

Our previous cytoarchitectural description ${ }^{10}$ of the human basal forebrain was used to delineate subregions of the Ch4: anteromedial, anterolateral, dorsal and ventral intermediate, and posterior. The volume of the Ch4, neuronal number, and mean diameter were determined in a complete series of Nissl stained sections with the aid of an NEC computer and Magellan software. ${ }^{10}$ Computer reconstructions of the $\mathrm{Ch} 4$ nucleus were created for each case to assess the distribution of cell loss. The volumes of the cerebral hemispheres, cortical grey, subcortical grey, and white matter (subcortical white matter, internal and external capsules, corpus callosum and anterior commissure) were determined by point counting on complete series of black and white photographs of 3 $\mathrm{mm}$ brain slices, as previously described. ${ }^{12}$ The volumes of the cerebral hemispheres, cortical grey matter, and white matter were then compared with Ch4 cell number using regression analysis.

Quantitative results were derived with observers blind to the clinical diagnosis of the case, including memory status. Data from all brain regions were acquired independently. Patient differences were assessed using analysis of variance (ANOVA) with protected $t$ tests. Discriminant analysis was used to determine deviations of individual cases from diagnostic groupings on the basis of $\mathrm{Ch} 4$ cell number and cortical volumes. Relations between patient variables and $\mathrm{Ch} 4$ cell number were tested using regression analysis. Quantitative results from two observers were not significantly different in $t$ tests $(\mathrm{P}>0.05)$. No $\mathrm{Ch} 4$ variable was significantly correlated to patients' postmortem delay, sex, or cause of death.

\section{Results}

In the vicinity of the Ch4, gross morphological changes including forebrain atrophy, ventricular dilatation, and glial scarring disrupted the normal forebrain architecture and gave an irregular concave appearance to the base of the brain in all cases of Wernicke's encephalopathy. In these cases, the $\mathrm{Ch} 4$ nucleus appeared as a narrower, denser band of cells along the basal surface of the brain compared with alcoholic patients without Wernicke's encephalopathy and controls. Accumulation of lipofuscin pigment was pronounced in all alcoholic patients, particularly cases WE1, WE2, WE5, and KP3.

In all cases, alcoholic and non-alcoholic, most Nissl stained magnocellular neurons were calbindin immunoreactive $(>95 \%, \mathrm{P}<0.05)$. Reduced numbers of calbindin positive fibres within Ch4 cell groups was seen in several cases of Wernicke's encephalopathy, most notably WE4 and WE5, and cases of Korsakoff's psychosis, KP3 and KP4. Comparison of cell size of $\mathrm{Ch} 4$ neurons disclosed no difference in the mean cell diameters between Korsakoff's psychosis, Wernicke's encephalopathy, alcoholic, and non-alcoholic control groups (table), although mean cell diameters in cases WE5 and KP4 were significantly below group means.

There was no significant difference in cell number between alcoholic controls and nonalcoholic age matched controls ( $P>0.05$, ANOVA; figure, A). The mean number of $\mathrm{Ch} 4$ neurons in the Korsakoff's psychosis and Wernicke's encephalopathy groups differed significantly from non-alcoholic control groups $(\mathrm{P}<0.02$, Fisher's protected $t$ test; figure, $\mathrm{A})$, with the Wernicke's encephalopathy mean $24 \%$ and the Korsakoff's psychosis mean $21 \%$ below that of controls. The Wernicke's encephalopathy group was significantly below alcoholic controls $(-21 \%, \mathrm{P}<0.02)$, but the difference did not reach significance for the Korsakoff's psychosis group $(-18 \%, \mathrm{P}<0.06)$. There was substantial overlap between all groups. Discriminant analysis showed that on the basis of age and Ch 4 cell number, cases WE1 and KP1 were within control group ranges $\left(\mathrm{F}_{2,17}=4.6\right.$, $\mathrm{P}=0.026)$ and the alcoholic and non-alcoholic groups could not be distinguished $\left(\mathrm{F}_{2,17}=0.8\right.$, $\mathrm{P}=0.47$ )

A homogeneous reduction of cell number per $50 \mu \mathrm{m}$ section was noted in all subregions of the Ch4 indicating that no focal loss of neurons occurred in any patients with Wernicke's encephalopathy or Korsakoff's psychosis. Despite lower neuronal counts in some patients with Wernicke's encephalopathy and Korsakoff's psychosis compared with controls, no consistent decrease in packing density was 



(A) Estimated number of Ch4 neurons plotted for each group. Columns represent mean values for non-alcoholic controls, alcoholic patients, and patients with Wernicke's encephalopathy (WE) and Korsakoff's psychosis (KP). Means for Korsakoff's psychosis and Wernicke's encephalopathy did not differ significantly (P>0.05), but both thiamine deficient groups differed significantly from alcoholic and non-alcoholic controls (controls, Wernicke's encephalopathy, $P<0.007$; controls, Korsakoff's psychosis, $P<0.023$; alcoholic controls, Wernicke's encephalopathy, $P<0.024$, alcoholic controls, Korsakoff's psychosis, $P<0.06)$. (B) Regression analysis of $\mathrm{Ch} 4$ cell number and age. Regression lines and 95\% confidence intervals are shown for controls (non-alcoholic and alcoholic). For controls, $r^{2}=0.554 ; P<0.0035$, and when thiamine deficient groups are included $r^{2}=0.23 P<0.04$, suggesting that these cases deviate from the expected Ch4 cell numberlage relation. (C) Regression analysis of Ch4 cell number and cortical volume, shown here for all groups together. Dotted lines show 95\% confidence intervals. A single regression relates the two variables for all groups $\left(r^{2}=0.385\right.$, $P<0.002)$, indicating that the Ch4 cell number is linked to the volume of the cortex.

noted. An increased cell density was seen in most alcoholic cases, with the exception of cases of Wernicke's encephalopathy, WE5 and KP4. Increased density was the only change noted in most cases in the non-WE alcoholic group, with gliosis accompanying density changes, particularly case A5.

As previously noted, ${ }^{11} \mathrm{Ch} 4$ cell number in alcoholic and non-alcoholic controls was significantly correlated with age $\left(r^{2}=0.55\right.$, $\mathrm{P}<0.0035)$. A significant correlation was also seen when all cases were considered $\left(r^{2}=0.23\right.$, $\mathrm{P}<0.04)$. The age/Ch4 relation for Wernicke's encephalopathy and Korsakoff's psychosis cases KP3, WE3, WE4, and WE5 (figure, B) was significantly different from controls on discriminant analysis $\left(\mathrm{F}_{2,17}=4.6, \mathrm{P}=0.026\right)$.

Measurement of cerebral volumes in controls and alcoholic groups disclosed atrophy of white matter but not cortical grey matter with high alcohol intake (table). ${ }^{8}$ Both cerebral hemisphere and cortical grey matter volumes were significantly correlated with $\mathrm{Ch} 4$ cell number in alcoholic and non-alcoholic control groups, and this correlation was preserved in the Wernicke's encephalopathy and Korsakoff's psychosis groups $\left(r^{2}=0.385, \mathrm{P}<0.002\right.$; all cases together; figure, $\mathrm{C}$ ).

\section{Discussion}

The present study examined $\mathrm{Ch} 4$ cell number and size in controls, alcoholic patients without neurological complications, and alcoholic patients with Wernicke's encephalopathy. Our aim was to test the hypotheses that (1) Ch4 cell loss distinguishes the amnesic from the nonamnesic patients with Wernicke's encephalopathy and (2) Ch4 cell loss correlates with alcohol intake. Our results corroborate earlier reports that cell number remains unchanged in alcoholic patients without Wernicke's encephalopathy. ${ }^{15}$ The data also show that Ch4 cell number is reduced in patients with Wernicke's encephalopathy both with and without Korsakoff's psychosis, consistent with our previous finding of neurofibrillary tangles in the $\mathrm{Ch} 4$ of alcoholic patients with Wernicke's encephalopathy. ${ }^{4}$ Evidence of cell loss in non-amnesic patients with Wernicke's encephalopathy, however, refutes previous, as yet unchallenged, conclusions of a causal relation between reduced Ch4 cell number and memory loss. ${ }^{6}$ These results cast doubt on the notion that alcoholic Korsakoff's psychosis is a "basal forebrain" amnesia. ${ }^{16}$

Despite the continued popularity of the cholinergic hypothesis, interpretation of the Ch4 deficit in Korsakoff's psychosis has been confounded by several factors, including controversial clinical definitions of the amnesia. ${ }^{17}$ Recently devised operational criteria strictly define Korsakoff's psychosis as a stable amnesic syndrome occurring in patients with Wernicke's encephalopathy against a background of an intact sensorium. ${ }^{9}$ Using this definition, patients with Wernicke's encephalopathy with and without Korsakoff's psychosis can be classified with a high degree of certainty. ${ }^{9}$ Whereas several of the patients with Wernicke's encephalopathy in the present study exhibited acute confusion and disorientation and mild memory impairment, memory functions were restored when the Wernicke's encephalopathy episode cleared. Thus two groups, both of which showed the characteristic neuropathological lesions of Wernicke's encephalopathy, could be distinguished clinically: (1) patients with Wernicke's encephalopathy with classic neurological signs and (2) patients with Wernicke's encephalopathy with neurological signs accompanied by a stable, 
persistent amnesia. By contrast, previous studies of the $\mathrm{Ch} 4$ have not included non-amnesic patients with Wernicke's encephalopathy, ${ }^{5} \mathrm{de}-$ spite documentation that Korsakoff's psychosis is not an inevitable outcome of Wernicke's encephalopathy. ${ }^{9}$ Earlier studies also included an alcoholic patient group with Korsakoff's psychosis but without Wernicke's encephalopathy ${ }^{5}$ that could not be distinguished in our necropsy series of more than 4000 cases. $^{9}$ We suggest that if current operational criteria were employed, all such alcoholic patients with Korsakoff's psychosis would show evidence of Wernicke's encephalopathy pathology.

Although Ch4 cell loss cannot account for the amnesia of Korsakoff's psychosis, lower $\mathrm{Ch} 4$ cell number may contribute to cognitive dysfunction in patients with Wernicke's encephalopathy, both with and without Korsakoff's psychosis. ${ }^{3-18}$ In the present study, alcoholic patients with Wernicke's encephalopathy but without Korsakoff's psychosis exhibited mild cognitive impairment, including intermittent disorientation and frontal lobe signs such as impaired planning and abstraction. Memory difficulties, such as reduced digit span, were noted in three cases and case WE5 showed specific deficits in attention and difficulty in the inhibition of irrelevant information. This last case (showing the greatest Ch4 cell loss) provides support for a correlation between cholinergic lesions and pronounced attentional deficits but not memory loss itself, as has been postulated from animal models. ${ }^{19}$ Thus $\mathrm{Ch} 4$ cell loss may be relevant to the clinical picture of Korsakoff's psychosis, which includes deficits in sustained attention and sensitivity to interference ${ }^{20}$ in addition to the striking anterograde amnesia.

The mild Ch4 cell loss in chronic Wernicke's encephalopathy (with or without Korsakoff's psychosis), reduced cell size in only two cases (WE5 and KP4), with the most extensive cell loss and the absence of cell loss in acute Wernicke's encephalopathy is in stark contrast to the gross necrotic lesions in the hypothalamus and thalamus of these patients. Several scenarios may account for the difference in severity between basal forebrain and diencephalic pathology. As the Ch4 has a substantial projection to the mediodorsal nucleus of the thalamus, a key site of pathology in Wernicke's encephalopathy, ${ }^{1-3}$ cell loss may occur via a retrograde mechanism rather than via direct insult.

The correlation between cortical atrophy and $\mathrm{Ch} 4$ cell number may also reflect an anterograde or retrograde degeneration. Primary changes to parenchymal arterioles, consisting of endothelial swelling, dilatation, smooth muscle and elastin degeneration, and petechial haemorrhages have been noted in thiamine deficiency. ${ }^{21}$ The cholinergic forebrain nuclei projections have been shown to contact parenchymal blood vessels, with a putative role in regulation of local cerebral blood flow. ${ }^{22}$ Damage to the thalamic or cortical vasculature as a primary degenerative mechanism in Wernicke's encephalopathy may result in secondary loss of $\mathrm{Ch} 4$ cells via neurofibrillary tangle formation. In this light, it is interesting to note that neurofibrillary tangles in the $\mathrm{Ch} 4$ have been noted ipsilateral to a cerebral infarct. ${ }^{23}$

There has been considerable debate as to the relative contributions of ethanol intake and nutritional deficiency to alcoholic brain damage. Most examples of alcoholic dementia may be attributable to metabolic or nutritional complications of alcoholism; ${ }^{24}$ however, thiamine deficiency must apparently occur on a background of high alcohol intake for development of the full Wernicke-Korsakoff syndrome. ${ }^{25}$ Despite histories of high alcohol intake (both amount consumed per day and lifetime intake) in all alcoholic patients studied, Loss of Ch4 cells occurred only in those with evidence of long term dietary deficiency and no loss was seen after a single acute episode of Wernicke's encephalopathy. This corroborates our previous work showing neurofibrillary tangle formation only in alcoholic patients with chronic Wernicke's encephalopathy ${ }^{4}$ and further supports a relation between thiamine deficiency, but not alcohol intake alone, and $\mathrm{Ch} 4$ cell loss. By contrast, rat models of chronic ethanol intoxication show a correlation of memory dysfunction with basal forebrain cell loss or reduced cholinergic input to the hippocampus, ${ }^{26}{ }^{27}$ with cholinergic rich transplants improving performance on behavioural tests. ${ }^{27}$ These studies suggest that, at least in rodents, alcohol is a primary neurotoxin that can cause memory dysfunction. To reconcile human studies with those in rats, it could be conjectured that the $\mathrm{Ch} 4$ homologue in rats is more vulnerable to alcohol toxicity than is the human $\mathrm{Ch} 4$. Indeed, $\mathrm{Ch} 4$ neurochemistry differs between rodents, non-human primates, and humans. ${ }^{13}{ }^{28}$ However, as ethanol reduces thiamine absorption, malnutrition may be an unrecognised confounder in studies of alcohol fed rats. ${ }^{1}$ Consistent with this notion, experimentally induced thiamine deficiency can produce a cholinergic deficit in rats. ${ }^{29}$

\section{Conclusion}

Although cholinergic involvement in the amnesic syndrome is widely cited in the literature on Korsakoff's psychosis, the studies supporting the hypothesis have lacked appropriate controls. ${ }^{56}$ Our results corroborate previous work showing Ch4 cell loss in Korsakoff's psychosis; however, because a similar lesion was also seen in non-amnesic Wernicke's encephalopathy, we cannot conclude that Ch4 loss constitutes the definitive anatomical substrate for the amnesia. These findings contribute important information that considerably alters the general conclusions of previous postmortem studies of alcoholic Korsakoff's psychosis. Nevertheless, if it is assumed that $\mathrm{Ch} 4$ pathology is not silent in alcoholic patients with Wernicke's encephalopathy, a component of their cognitive impairment, such as attentional deficits, may be attributable to cholinergic cell loss. Our results do not support a critical role for the Ch4 in memory loss in Korsakoff's psychosis, but provide further support for a more circumscribed role for the Ch4 in attention. ${ }^{19}{ }^{30}$ This 
finding has direct clinical relevance as treatment of patients with Korsakoff's psychosis with cholinergic replacement therapy (tetrahydroaminoacridine) has been suggested. ${ }^{31}$

We thank the staff at the Neuropathology Unit, University of Sydney, for preparation of material for diagnosis, Professor Clive Harper for neuropathological diagnoses, and Dr Robert Heard and staff at the Neuropsychology Unit for assisting with acquisition of the neuropsychological data. We thank Dr Antony Harding and Heidi Cartwright for assistance with histologica material. This study was supported by the National Medica Health and Research Council of Australia, the Government Employees' Fund for Medical Research, and a grant from the National Institute on Alcohol Abuse and Alcoholism (Grant 931260).

1 Victor M, Adams RD, Collins GH. The Wernicke-Korsakoff syndrome and related neurologic disorders due to alcoholism and malnutrition. 2nd ed. Philadelphia: F A Davies Company, 1989.

2 Harper CG, Kril JJ. Neuropathology of alcoholism. Alcohol and Alcoholism 1990;25:207-16.

3 Torvik A. Topographic distribution and severity of brain lesions in Wernicke's encephalopathy. Clin Neuropathol 1987;6:25-9.

4 Cullen KM, Halliday GM. Tangles in chronic alcoholics. Neuropathol Appl Neurobiol 1995;21:312-8.

5 Arendt T, Bruckner M, Bigl V, Marcova L. Dendritic reorganisation in the basal forebrain under degenerative conditions and its defects in Alzheimers disease. 2. Ageing, Korsakoff's disease, Parkinson's disease, and Alzheimer's sakoff's disease, Parkinson's disease, and
disease. f Comp Neurol 1995;351:189-222.

6 Arendt T, Bigl V, Arendt A, Tennstedt A. Loss of neurons in the nucleus basalis of Meynert in Alzheimer's disease, paralysis agitans and Korsakoff's disease. Acta Neuropatho 1983;61:101-8.

7 Mesulam M-M, Geula C. Nucleus basalis (Ch4) and cortical cholinergic innervation in the human brain: observations based on the distribution of acetylcholinesterase and choline acetyl transferase. 7 Comp Neurol 1988;275:21640.

8 Kril JJ, Halliday GM, Svoboda MD, Cartwright H. The cerebral cortex is damaged in chronic alcoholics. Neuroscience 1997;79:983-98.

9 Caine D, Halliday GM, Kril J, Harper CG. Operational criteria for the classification of chronic alcoholics for research teria for the classification of chronic alcoholics for research and

10 Halliday GM, Cullen KM, Cairns MJ. Quantitation and three-dimensional reconstruction of Ch4 nucleus in the human basal forebrain. Synapse 1993:15:1-16.

11 Cullen KM, Halliday GM, Double KL, Brooks WS, Creasy $\mathrm{H}$, Broe GA. Cell loss in the nucleus basalis is related to regional cortical atrophy in Alzheimer's disease. Neuroscience 1997;78:641-52.

12 Double KL, Halliday GM, Kril JJ, et al. Topography of brain atrophy during normal aging and Alzheimer's disease. Neurobiol Aging 1995;17:513-21.
13 Geula C, Schatz CR, Mesulam M-M. Differential localization of NADPH-diaphorase and calbindin-D28k within the cholinergic neurons of the basal forebrain, striatum and brainstem in the rat, monkey, baboon and human. Neuroscience 1993;54:461-76.

14 Cullen KM, Halliday GM, Cartwright H, Kril JJ. Improved selectivity and sensitivity in the visualization of neurofibrillary tangles, plaques and neuropil threads. Neurodegeneration 1996;5:177-87.

15 Akai J, Akai K. Neuropathological study of the nucleus basalis of Meynert in alcoholic dementia. Fapanese fournal of Alcohol Studies and Drug Dependence 1989;24:80-8.

16 Butters N. Alcoholic Korsakoff's syndrome: some unresolved issues concerning etiology, neuropathology, and colved issues concerning etiology, neuropathology, and

17 Lishman WA. Alcoholic dementia: a hypothesis. Lancet 1986;ii:1 184-6.

18 Jacobson RR, Lishman WA. Selective memory loss and global intellectual deficits in alcoholic Korsakoff's syndrome. Psychol Med 1987;17:649-55.

19 Voytko ML, Olton DS, Richardson RT, Gorman LK, Tobin JR, Price DL. Basal forebrain lesions in monkeys disrupt attention but not learning and memory. $\mathcal{F}$ Neurosci 1994;14:167-86.

20 Salmon DP, Butters N. The etiology and neuropathology of alcoholic Korsakoff's syndrome: some evidence for the role of the basal forebrain. In: Galanter $M$, ed. Recent of the basal forebrain. In: Galanter M, ed. Recent developin.

21 Okeda R, Taki K, Ikari R, Funata N. Vascular changes in acute Wernicke's encephalopathy. Acta Neuropathol 1995; 89:420-4

22 Linville DG, Williams S, Arneric SP. Basal forebrain control of cortical cerebral blood flow is independent of local cortical neurons. Brain Res 1993;622:26-34.

23 Kato T, Hirano A, Katagiri T, Sasaki H, Yamada S. Neurofibrillary tangle formation in the nucleus basalis of Meynert ipsilateral to a massive cerebral infarct. Ann Neurol 1988;23:620-3.

24 Victor M. Alcoholic dementia. Can 7 Neurosci 1994;21:8899.

25 Freund G. Chronic central nervous system toxicity of alcohol. Annu Rev Pharmacol Toxicol 1973;13:217-27.

26 Melis F. Chronic ethanol consumption in rats: correlation between memory performance and hippocampal acetylcholine release in vivo. Neuroscience 1996;74:155-9.

27 Arendt T, Bigl V, Schugens MM. Ethanol as a neurotoxin - a model of the syndrome of cholinergic deafferentation of the cortical mantle. Brain Dysfunction

Walker LC, Koliastos VE, Kitt CA, Richardson RT, Rökaeus A, Price DL. Peptidergic neurons in the basal forebrain magnocellular complex of the rhesus monkey. 7 Comp Neurol 1989;280:272-82.

29 Butterworth R. Neurotransmitter function in thiaminedeficient encephalopathy. Neurochem Int 1982;4:449-64.

30 Blokland A. Acetylcholine: a neurotransmitter for learning and memory? Brain Res Rev 1996;21:285-300.

31 Nevo I, Hamon M. Neurotransmitter and neuromodulatory mechanisms involved in alcohol abuse and alcoholism. Neurochem Int 1995;26:305-36. 